\title{
A New Architecture Design of Industrial Networks
}

\author{
Shan Zhu ${ }^{1, a}$, Shi Xiao ${ }^{1, b, *}$ \\ ${ }^{1}$ Institute of Electronic \& Electrical Engineering, Wuhan Textile University, Hubei430072, China \\ ${ }^{2}$ Department of Communication engineering, Wuhan Textile University, Hubei430072, China \\ a zs1004ypf@163.com, ${ }^{b}$ xiaoshi@wtu.edu.cn \\ Correspondence should be addressed to Shi Xiao; xiaoshi@wtu.edu.cn
}

Keywords: industrial networks, intelligent manufacturing, low-latency, high-reliability, TCP/IP model

\begin{abstract}
Industrial networks, regarded as the core of intelligent manufacturing, mostly are implemented to support various monitoring applications in industrial environments. As such, the low-latency and high-reliability are the vital metrics especially under heavy loads. However, the existing conventional TCP/IP network fails to provide real-time communications for data transmitted between devices and controllers. To satisfy strict restrictions on latency and reliability of industrial communication, this paper designs a new architecture based on the existing TCP/IP model and a hybrid scheme. Special applications in industrial networks, such as industrial control information transmission, could be transmitted along the special channels by using the proposed method to guarantee their real-time. Finally, the simulation results demonstrate that the proposed architecture exhibits great performance in real-time and reliability.
\end{abstract}

\section{Introduction}

In the era of "industry 4.0" and "China Intelligent Manufacturing", the industrial Internet has become a hot topic, focusing on the development of fully-automated "smart" factories. As the core of intelligent manufacturing, industrial networks play a key role in upgrading of the manufacturing sector to improve innovation ability. However, industrial networks are deployed to connect end productive facilities, and mostly required to support those monitoring applications for production environments [1-2]. For this reason, to meet the demands of special applications in industrial networks, such as industrial control information transmission, industrial networks put forward stringent requirements on latency and reliability. Due to the fact that the traditional TCP/IP mechanism is designed for commercial networks, there would be obvious defects if conventional TCP/IP mechanism was directly applied to industry. Therefore, we need to do in-depth research on industrial networks.

Despite differences between industrial and commercial networks, the use of TCP/IP mechanism presents several advantages, especially the low cost based on the mature technologies. To make the best of these advantages applied in industrial applications, some modifications or extensions are needed on the existing TCP/IP mechanism to satisfy the specific requirements of industrial networks. Progresses have been made in research on the problem of industrial networks from the theoretical and implementation side, but due to the strict requirements on latency and reliability, so far industrial communication systems do not have an optimal solution yet. As a result, this paper introduces a different approach to address the challenges for industrial applications by modifying the existing protocol architecture of TCP/IP mechanism in terms of the universal applicability. An optimized architecture is proposed in this paper to meet the specific needs of industrial networks, which are supposed to meet latency and reliability requirements.Simulation results are demonstrated to evaluate the proposed architecture design for industrial applications. 


\section{Overview}

\subsection{Requirements of Industrial Networks}

A simple network of representative application [3] is shown in Figure 1. Industrial networks must meet the special needs of network performance for industrial applications. The network performance requirements can be summarized as follows.

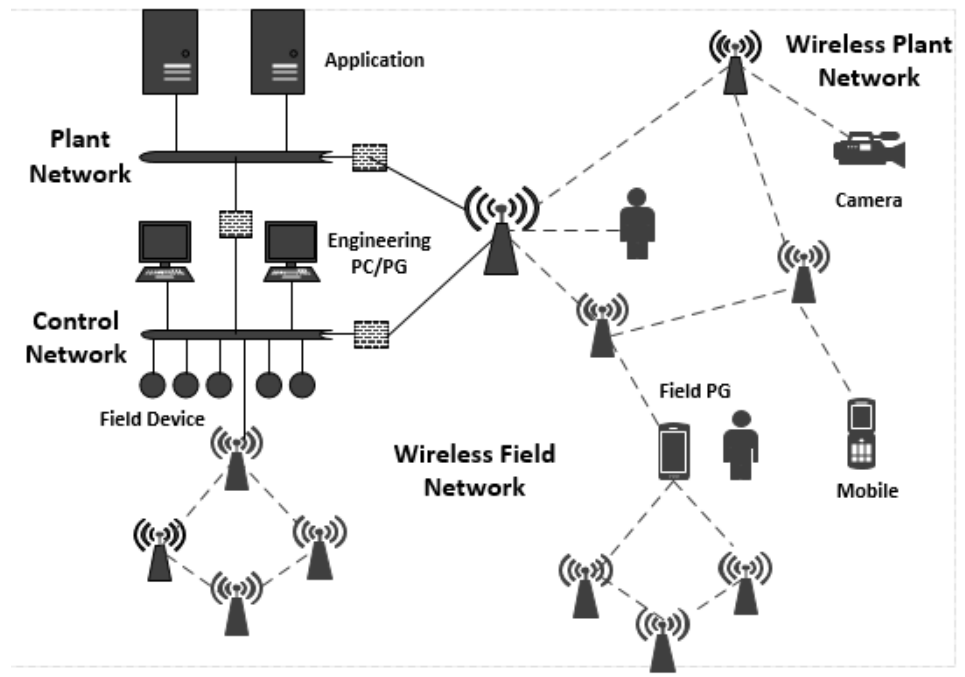

Figure1: Example of Representative Application

\subsubsection{Real -Time Requirements}

Real-time indicates the ability of a system to process external events within a certain time. The operation speed of equipments and production processes are so fast that the time cost of data transmission, procession and response are required as little as possible. The real-time of the network is the primary concern of many industrial users because it is the most important performance index of network systems. Different industrial applications have different real-time requirements. The real-time requirements in different industrial applications are given in Table 1.

Table1 Different real-time requirements in different applications

\begin{tabular}{|c|c|c|c|}
\hline Applications & Domains & Real-time & $\begin{array}{c}\text { Typical } \\
\text { Grace time }\end{array}$ \\
\hline Non real-time control & $\begin{array}{c}\text { Building, automation } \\
\text { management }\end{array}$ & Second \\
\hline Process control & $\begin{array}{c}\text { Metallurgy, chemical, power } \\
\text { plant }\end{array}$ & Millisecond & $20 \mathrm{~ms}$ \\
\hline Motion control & Manufacturing, robots & Microsecond & $2 \mathrm{~ms}$ \\
\hline
\end{tabular}

The IP communication network enables people to interact with information on the web, while industrial control networks are more involved in information interaction between physical equipments. According to demands of different control task, data streams of production process have potential delay tolerance limits. Thus, compared with general real-time applications such as multimedia real-time application which focuses on the network average delay performance, industrial applications are more concerned about whether the end-to-end latency of all the data flows meets the requirements of control tasks.

\subsubsection{Reliability Requirements}

As the core part of modern control systems, the reliability performance of industrial networks directly affects the reliability of control systems. The reliability requirement of network in industrial application is embodied in two aspects. One is the minimum network reliability, which reflects the ability of the network to operate without fault at specified time. The second is the interruption time of network which systems can tolerate. When the failure of network systems occurs, its recovery 
time should be less than its grace time. The grace time in different industrial applications are shown in Table 1.

\subsection{Problems of Current Research on Industrial Networks Architecture}

The conventional Transmission Control Protocol/ Internet Protocol (TCP/IP) model consists of four layers, which are physical, network, transport and application layers, respectively [4-6].In spite of the fact that TCP/IP is still used throughout the Internet, the large amount of delay and overhead in the transmitted data results in the failure to guarantee real-time and reliable communication for industrial networks. Thus, many researchers try to simplify the protocol architecture. For example, according to the reduced model defined in [7], the proposed architecture is only made up of MAC layer and PHY layer. In that paper, a synchronization mechanism is proposed in which the durations of transmissions and retransmissions are fixed scheduled, thus the strict constraints could be achieved. However, being lack of flexibility, these changes of architecture are only valid for periodic data transmission of industrial control systems. In fact, industrial networks transmit not only periodically sampled data, but also non-periodic data that may occur at any time such as alarm conditions.

\section{New Architecture}

Due to the fact that industrial networks must be measured by their real-time capability and high efficiency under the heavy load, a preliminary architecture is proposed, focusing on achieving the low-latency and high-reliability for industrial applications.

Considering the universal applicability, the proposed architecture is based on TCP/IP. Meanwhile, to satisfy the specific requirements of industrial applications, RE-ATM is introduced to transmit real-time data packets in the proposed architecture, as shown in Figure 2. While standard device data transmitted in industrial levels and IT levels are generally used TCP/IP protocol in standard channel, RT channels are specially provided for real-time data such as control signal in industrial applications by the RE-ATM method.

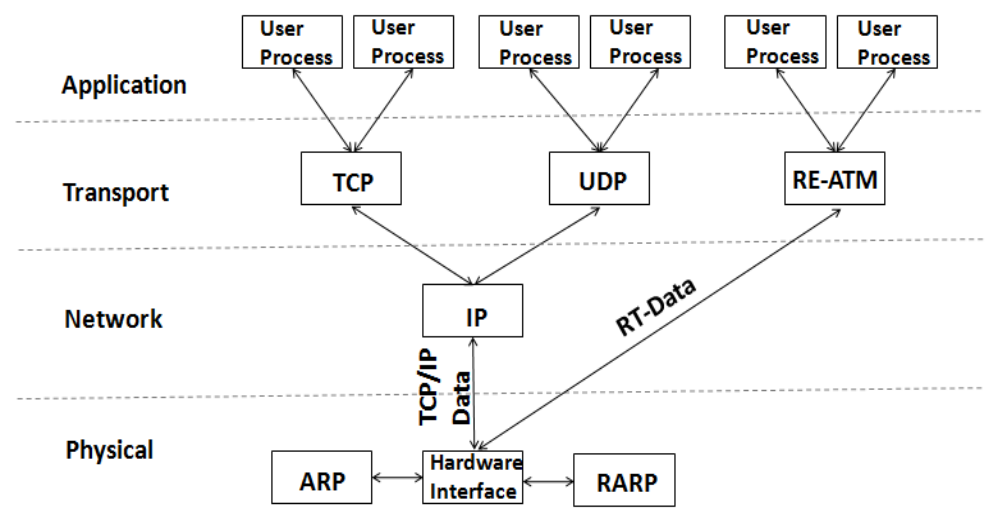

Figure 2: Network stack configurations

In the proposed architecture, the use of protocols for different hierarchies is based on type of data that need to be sent. For example, if application data is TCP/IP data, it means that these data do not have strict real-time requirement. Thus, these data use TCP or UDP protocol in the transport layer, which is achieved by the type of service and protocol defined in the IP header. At the same time, if application data is RT-data such as control information sent between Programmable Logic Controller (PLC) I/O controller and I/O device, these data have strong deterministic and real-time requirements [8-12]. As such, these data use RE-ATM along the special real-time channel.

\section{Simulation Results}

In this section, simulation results are presented to evaluate the proposed architecture compared to the existing TCP/IP architecture. To verify these improvements in the proposed architecture, simulation scenario is based on NS-2 as follows. For this example, the data rate is set to the range of $5 \mathrm{Kbps}$ to $295 \mathrm{Kbps}$, and the step is $10 \mathrm{Kbps}$. The simulation running time is 10 s. While standard data 
starts at $0.5 \mathrm{~s}$ and ends at $9.5 \mathrm{~s}$, real-time data starts at $1 \mathrm{~s}$ and ends at $9 \mathrm{~s}$. The size of standard data is 210 bytes and the real-time data packet length is 4 bytes.

With the growth of data rate, Figure 3 demonstrates that data delays of the proposed architecture show a slow rise; however, data delays of the existing TCP/TP architecture increase quickly when the data rate is greater than 250Kbps. In addition, Figure 4 illustrates that using the proposed architecture exhibits better efficiency than the existing TCP/IP model under heavy loads. Consequently, simulation results verify that the designed architecture for industrial applications could provide outstanding performance even under heavy traffic.

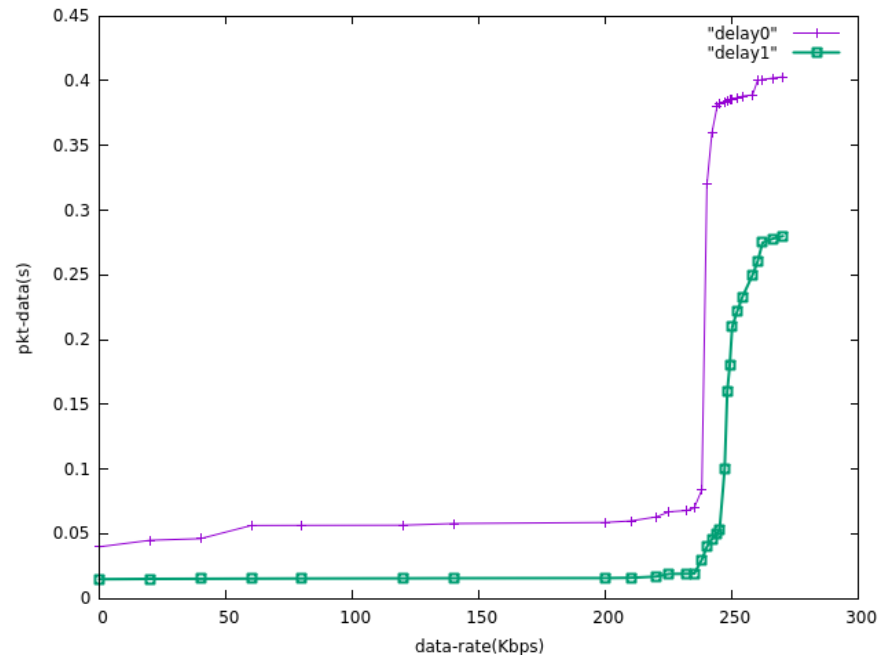

Figure 3: Data delay

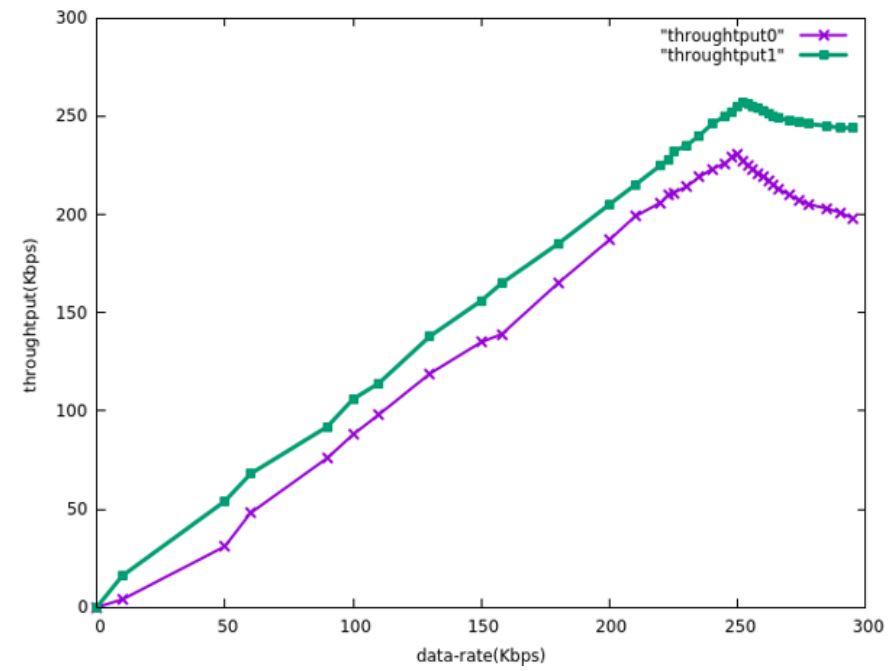

Figure 4: Data throughput

\section{Conclusion}

This paper presents a new architecture design of communication system which displays a lowlatency and high-reliability performance, making it particularly appropriate for industrial applications. The proposed architecture is based on the existing TCP/IP model and a hybrid scheme. Furthermore, the data transmitted between devices nodes and controller nodes show good real-time and high efficiency under heavy loads by using the special scheduling.

\section{Acknowledgements}

This work is supported in part by the Youth Project of Hubei Province under Grant no.17Q091, Undergraduate Programs for Innovation of Hubei Province under Grant no.201613240004 and Fund 
Projects of Wuhan Textile University under Grant no.155028.

\section{References}

[1] B. Galloway and G. P. Hancke, "Introduction to Industrial Control Networks", IEEE Communications Surveys \& Tutorials, 2013 , 15 (2) :860-880

[2] Qiang Liu et al, "A Real-time and Efficient MAC Protocol for Smart Grid Wireless Communications,” in International Journal of Distributed Sensor Networks, vol. 2014.

[3] J. Song, S. Han, A. K. Mok et al., "WirelessHART: applying wireless technology in real-time industrial process control," in Proceedings of the IEEE Real-Time and Embedded Technology and Applications Symposium (RTAS '08), pp. 377-386, St. Louis,Mo, USA, April 2008.

[4] W.Richard Stevens, TCP/IP Illustrated, volume 1: The Protocols [M], vol. 2000, pp. 1-14, Apr.2016.

[5] J.-D. Decotignie, “A perspective on Ethernet-TCP/IP as a field bus," in IFAC international conference on field bus systems and their application, Nov 2001, pp. 138-143

[6] D.J Wang et al, Siemens Industrial Network: Communication Technology Illustrated [M], vol.2012, pp. 46-49, Mar. 2014.

[7] M Weiner ,M Jorgovanovic , A Sahai ,B Nikolie.” Design of a Low-Latency, High-Reliability Wireless Communication System for Control Applications," IEEE International Conference on Communications , 2014 :3829-3835

[8] G Durisi , T Koch , P Popovski.”'Toward Massive, Ultrareliable, and Low-Latency Wireless Communication With Short Packets,” in Proceedings of the IEEE , 2016 , 104 (9) :1711-1726.

[9] T. Sauter, "The three generations of field-level networks - evolution and compatibility issues," IEEE Transactions on Industrial Electronics, vol. 57, no. 11, pp. 3585-3595, Nov 2010.

[10] J.-P. Thomesse, "Fieldbus technology in industrial automation," Proceedings of the IEEE, vol. 93, no. 6, pp. 1073-1101, June 2005.

[11] F. li Lian, J. Moyne, and D. Tilbury, "Network design considerations for distributed control systems," IEEE Transactions on Control Systems Technology, vol. 10, no. 2, pp. 297-307, Mar 2002.

[12] J. R. Moyne and D. M. Tilbury, "The emergence of industrial control networks for manufacturing control, diagnostics, and safety data," Proceedings of the IEEE, vol. 95, no. 1, pp. 29-47, Jan 2007. 